\title{
Genotyping of amino acid-producing Corynebacterium glutamicum strains based on multi-locus sequence typing (MLST) scheme
}

Junjie Yang ${ }^{1,2,4}$, Yimeng Kong ${ }^{1,3}$ and Sheng Yang ${ }^{1,2,4^{*}}$

\begin{abstract}
Background: Confusing parental information may hinder to dissect mechanisms of amino acid hyper-producing Corynebacterium glutamicum strains. Thus, an efficient method for genotyping of the C. glutamicum is heavily called.

Results: Multi-locus sequence typing (MLST) is currently the most popular molecular typing technique. But currently this method is not available for C. glutamicum. In this study, a MLST scheme was established based on sequences of seven housekeeping genes, for genotyping of C. glutamicum. The MLST method performed an efficient discrimination of 17 strains and helps to understand the population structure of this bacterium.
\end{abstract}

Conclusions: This work has expanded the MLST method to C. glutamicum and developed an efficient technique to discriminate strains of uncertain origin.

Keywords: Corynebacterium glutamicum; Multi-locus sequence typing; Amino acid producing; Corynebacterium crenatum; 165 rDNA

\section{Background}

Non-spore-forming Gram-positive bacteria Corynebacterium glutamicum are widely used in amino acid production industry with numerous ideal attributes [1,2]. Due to roughly identical $16 \mathrm{~S}$ rDNA sequences, many independent strains previously regarded as Brevibacterium and Corynebacterium species in the Corynebacteriaceae family have been categorized into the C. glutamicum species, especially Brevibacterium lactofermentum, Brevibacterium flavum, and Corynebacterium acetoacidophilum [3-5]. Consequently, the C. glutamicum species dropped into a mixture of strains from diversified resources.

Clear genetic or phylogenetic information is occasionally missing after a long-term utilization and preservation. Confusing parental information may hinder to dissect

\footnotetext{
* Correspondence: syang@sibs.ac.cn

'Key Laboratory of Synthetic Biology, Institute of Plant Physiology and Ecology, Shanghai Institutes for Biological Sciences, Chinese Academy of Sciences, 300 Fenglin Road, Shanghai 200032, China

${ }^{2}$ Shanghai Research Center of Industrial Biotechnology, Shanghai 201201, China

Full list of author information is available at the end of the article
}

mechanisms of amino acid hyper-production in these strains. For example, although genome sequences are now available for several glutamate-hyper-producing strains, e.g., SCgG1, SCgG2, and Z188, detail typing and parental information of these strains were still absent. To our knowledge, only an identification method by phage sensitivity has been reported, but this method is not very convenient because of the experimental procedure for handling phages [6]. Thus, an efficient method for genotyping of the C. glutamicum is heavily called for in this field.

Compared with other molecular typing techniques, multi-locus sequence typing (MLST) is currently the most popular one $[7,8]$. MLST relies on allelic variants in conserved genes to calculate phylogenetic relationship of strains. Multiple housekeeping genes (usually seven genes) are examined in the analysis as their sequences are constrained and variations are nearly neutral. Each strain is then assigned with an allelic profile or sequence type (ST). The MLST method excels other molecular 
typing methods of being unambiguous and easily portable between laboratories.

To our knowledge, in the family of Corynebacteriaceae, MLST scheme is only developed in Corynebacterium diphtheriae. More than 70 STs were identified and fall into at least 11 groups of $C$. diphtheriae. Meanwhile, no ST is currently available for C. glutamicum. In order to have accurate typing techniques for C. glutamicum, we developed a MLST scheme, which would promote applied research of this amino acid-producing species.

\section{Methods}

The strains used in this study are listed in Table 1 [3-6,9-13]. Genome sequences of the strains used for MLST analysis include ATCC13032 [5,2,1], MB1 [14], R [15], ScCG1, ScCG2, ATCC14067 [16], S9114 [17], Z188, 'C. crenatum' MT. Primers 27F 5' -AGAGTTTGATCMTGGCTCAG-3' and 1492R 5'-TACGGYTACCTTGTTACGACTT-3' were used to identify $16 \mathrm{~S}$ rDNA sequences of the strains $[3,4]$. The retrieved $16 \mathrm{~S}$ rDNA sequences are almost identical, with a similarity $>99 \%$ compared with the sequence of the C. glutamicum ATCC 13032, indicating that all of the strains belong to C. glutamicum $[3,4]$.

Referring to the genotyping scheme in C. diphtheriae [7], another species belonging to the same genus, seven housekeeping genes of the C. glutamicum strains were selected for analysis, including atpA, dnaE, dnaK, fusA, rpoB, leuA, and odhA (Table 2). All the loci are single copy in the genome sequences, according to the BLAST analysis. Considering the possibility to meet the case when only crude amino acid fermentation samples are available, which would limit the efficiency of PCR reactions, approximately 300 400 bp length of the PCR amplicon in housekeeping genes was designed for downstream experiments and analysis (Table 2).

\section{Results and discussion}

The PCR amplicon size ranges from $318 \mathrm{bp}(l e u A)$ to 402 bp (fusA), with a mean size of 369 bp (Table 2, Additional file 1: Figure S1 and Dataset S1). Each locus of housekeeping gene alleles for comparison and identification has a length ranging from $150 \mathrm{bp}($ leuA) to $243 \mathrm{bp}$ (dnaK), with a mean length of $206 \mathrm{bp}$. All alleles for a given locus are of equal lengths and in a correct reading frame. The proportion of variation sites in each locus varies from $3 \%(o d h A)$ to $17 \%$ (leuA) (Table 2). Primer and allele sequences are listed in Table 2 and Additional file 1: Dataset S2.

The dendrogram was drawn from allelic profile data using the UPGMA (unweighted pair group method with arithmetic mean) method. The online tool (http://pubmlst. org/) of the PHYLIP suite programs and Phylodendron [18] were implemented for tree generation, output, and display. The dendrogram demonstrates that eight groups of the strains could be classified according to their genetic distinctions (Figure 1). Except ST4/ST5 and ST9/ST10, which share six alleles respectively, most of STs share no more than three alleles with each other (Table 1), indicating that the strains could be well separated. The next section briefly describes the eight classified groups.

Group 1 (ST\#1): The C. glutamicum type strain ATCC13032 is a member of this group. MB001 is a prophage-free variant of ATCC13032 with a 6\% reduced genome [14]. Consistently, it has the same ST with ATCC13032.

Group 2 (ST\#2): 'B. lactofermentum' ATCC13869 represents an independent ST, as expected.

Group 3 (ST\#3): 'C. acetoacidophilum' ATCC13870 also represents an independent ST.

Group 4 (ST\#4 and \#5): Although 'B. flavum' ATCC14067 and its derived strain ATCC21493 [13] contain different STs, in fact, there is only 1-bp difference (in odhA, resulting in Ala701Thr) among the seven analyzed genes. So, the classification is correct.

Group 5 (ST\#6): C. glutamicum R [15] represents an independent ST.

Group 6 (ST\#7): 'C. pekinense' AS1.299 and its derived strain AS1.563 $[9,10]$ contain the same ST and were consequently classified into the same group.

Group 7 (ST\#8): C. glutamicum 617 (SIIM B1) also represents an independent ST.

Group 8 (ST\#9 and \#10): Several 'B. tianjinese' and ' $C$. crenatum' strains, including both 'wild-type' strains $[11,12,19]$ and derived strains $[12,17,20]$, represent the same ST (ST\#9), so they are categorized in this group. The strain ' $C$. crenatum' MT also fall into the group. It has a different ST (ST\#10) from 'wild-type' strain AS1.542 with only one different locus.

Taken together, the MLST results reveal a correct typing of the related strains. We also tested a commercially obtained crude lysine sample and several amino acid-producing strains preserved in our laboratory. The crude lysine sample represented a MLST result same to 13032, so it would be produced by an ATCC 13032-derived strain. Three lysine-producing strains fall into group $1(\mathrm{ST} \# 1)$, group 2 (ST\#2), and group 6 (ST\#7), separately. A proline-producing strain falls into group 3 (ST\#3). These results suggest that the eight groups defined in this study could encompass most of the amino acid-producing strains for laboratory use.

The aim of this work was to expand the MLST method to C. glutamicum and develop an efficient technique to discriminate strains of uncertain origin. After a long-term preservation, activation, and fermentation, parental information of the amino acidproducing strains is easily confused. The MLST scheme developed in our study addresses the problem 


\begin{tabular}{|c|c|c|c|c|c|c|c|c|c|c|c|c|}
\hline \multirow[t]{2}{*}{ ST } & \multirow[t]{2}{*}{ Group } & \multirow[t]{2}{*}{ Strains } & \multirow[t]{2}{*}{ Synonym } & \multirow[t]{2}{*}{ Descriptions } & \multirow[t]{2}{*}{ Genome sequence $^{a}$} & \multicolumn{7}{|c|}{ Allelic profile } \\
\hline & & & & & & $\overline{\operatorname{atp} A}$ & dnaE & dnaK & fusA & leuA & odhA & $\overline{r p o B}$ \\
\hline & & ATCC13032 & & C. glutamicum type strain & NC_003450, NC_006958 & & & & & & & \\
\hline \multirow[t]{2}{*}{1} & 1 & MB001 & & Prophage-free variant of ATCC 13032 with a 6\% reduced genome & NC_022040 & 1 & 1 & 1 & 1 & 1 & 1 & 1 \\
\hline & & Crude sample & & A crude lysine sample, commercially obtained & & & & & & & & \\
\hline 2 & 2 & ATCC13869 & B. lactofermentum & 'Wild-type B. lactofermentum' & & 1 & 2 & 2 & 4 & 2 & 4 & 4 \\
\hline 3 & 3 & ATCC13870 & C. acetoacidophilum & 'Wild-type C. acetoacidophilum' & & 4 & 6 & 5 & 5 & 6 & 1 & 1 \\
\hline 4 & 4 & ATCC14067 & B. flavum & J. Bacteriol. 194 (3), 742-743 (2012) & AGQQ01000000 & 3 & 2 & 4 & 6 & 2 & 2 & 2 \\
\hline 5 & 4 & ATCC21493 & B. flavum & Producing arginine, derived from ATCC 14067 (SIIM B234) & & 3 & 2 & 4 & 6 & 2 & 5 & 2 \\
\hline 6 & 5 & $\mathrm{R}$ & & C. glutamicum isolated in Japan from a meadow soil sample & NC_009342 & 5 & 3 & 7 & 3 & 3 & 2 & 1 \\
\hline \multirow[t]{2}{*}{7} & 6 & AS1.299 & C. pekinense & 'Wild-type C. pekinense,' producing glutamate (=CICC 10119, SIIM B3) & & 2 & 5 & 3 & 5 & 4 & 3 & 3 \\
\hline & & AS1.563 & C. pekinense & (=CICC 10178, SIIM B165) producing lysine, derived from AS1.299 & & & & & & & & \\
\hline \multirow[t]{3}{*}{8} & 7 & 617 & & $\begin{array}{l}\text { A glutamate-producing strain previously used in China } \\
(=\text { CICC 10117, SIIM B1) }\end{array}$ & & 1 & 2 & 4 & 7 & 7 & 3 & 2 \\
\hline & & T6-13 & B. tianjinese & 'Wild-type B. tianjinese' (=CICC 20182, SIIM B226) & & & & & & & & \\
\hline & & SCgG1 & & Hyper-producing glutamate & NC_021351 & & & & & & & \\
\hline \multirow[t]{4}{*}{9} & 8 & $\mathrm{SCgG} 2$ & & Hyper-producing glutamate & NC_021352 & 5 & 4 & 6 & 2 & 5 & 3 & 1 \\
\hline & & S9114 & & A strain for industrial production of glutamate & AFYA01000000 & & & & & & & \\
\hline & & Z188 & & Hyper-producing glutamate & AKXP01000000 & & & & & & & \\
\hline & & AS1.542 & C. crenatum & 'Wild-type C. crenatum' (=CICC10124, SIIM B6) & & & & & & & & \\
\hline 10 & 8 & MT & C. crenatum & A mutant of AS1.542 & AQPS01000000 & 6 & 4 & 6 & 2 & 5 & 3 & 1 \\
\hline
\end{tabular}


Table 2 Primer sequence and characteristics of the seven loci used in MLST

\begin{tabular}{|c|c|c|c|c|c|c|c|}
\hline Gene & primer & Sequence $\left(5^{\prime}-3^{\prime}\right)$ & $\begin{array}{l}\text { Amplicon } \\
\text { size (bp) }\end{array}$ & $\begin{array}{l}\text { Amplicon bps (from-to) } \\
\text { in NC_006958.1 }\end{array}$ & $\begin{array}{l}\text { Allele } \\
\text { size (bp) }\end{array}$ & $\begin{array}{l}\text { Number of } \\
\text { distinct alleles }\end{array}$ & $\begin{array}{l}\text { Number of } \\
\text { polymorphic sites }\end{array}$ \\
\hline \multirow[t]{2}{*}{$\operatorname{atpA}$} & atpA_F & ATGTACCAGGGCAACCAC & 390 & $1277256-1277645$ & 240 & 6 & 13 \\
\hline & atpA_R & GGCAACCTTCTTCATACC & & & & & \\
\hline \multirow[t]{2}{*}{ dnaE } & dnaE_F & GGCGAAGGATATTCCGTTG & 366 & $2220485-2220120$ & 225 & 6 & 23 \\
\hline & dnaE_R & ACTTCCCCATCGCGGTTG & & & & & \\
\hline \multirow[t]{2}{*}{ dnak } & dnaK_F & CACCTCACAGGAAATCTC & 397 & 2959446-2959050 & 243 & 7 & 17 \\
\hline & dnaK_R & GGACTGGAACTTCTCTAC & & & & & \\
\hline \multirow[t]{2}{*}{ fusA } & fusA_F & CAAGGCAGCTATCCGTAAGATG & 402 & $524614-525015$ & 237 & 7 & 11 \\
\hline & fusA_R & ATGTTACCAGCGTGTGCAACC & & & & & \\
\hline \multirow[t]{2}{*}{ leuA } & leuA_F & CTGGTTCAGGCTCGTGAGCA & 318 & $267591-267274$ & 150 & 7 & 26 \\
\hline & leuA_R & GATCATTGGGTTCTCAGGA & & & & & \\
\hline \multirow[t]{2}{*}{ odhA } & odhA_F & CITGGCTGGGTCATGGAAGG & 327 & $1175355-1175681$ & 186 & 5 & 5 \\
\hline & odhA_R & CTGGGCATCGTGCCAGAAAC & & & & & \\
\hline \multirow[t]{2}{*}{ rpoB } & rpoB_F & TATGTGACCGCGGAGTTC & 357 & $513278-513634$ & 165 & 4 & 5 \\
\hline & rpoB_R & GAAACGCTCGGTGATCTG & & & & & \\
\hline
\end{tabular}

and proved to be an efficient way to distinguish them. According to our results, three glutamate-producing strains mentioned above (SCgG1, SCgG2, and Z188) would derive from T6-13 or AS1.542. Their common genetic origin serves a firm premise for downstream comparative genomic analysis to compare parental strains and their offsprings. According to our MLST result, a lysine-producing strain CICC20042 was found to show ST\#9 (including T6-13, S9114, AS1.542, etc.) (detailed data not shown). But according to the CICC database, CICC20042 is derived from AS1.563 (ST\#7, including AS1.299 and AS1.563). This suggests that confusion about the parental information might have happened.

Several strains previously regarded as Brevibacterium and Corynebacterium species had been classified to $C$. glutamicum $[3,4]$. Our analysis extends the conclusion and proposes that $C$. glutamicum should also contain several $C$. pekinense, B. tianjinese, and $C$. crenatum strains. The 'wild-type' strains of $B$. tianjinese and $C$. crenatum exhibit the same ST. Both of them are highly sensitive to specific phages [6], supporting a close relationship of these strains. A brief comparative genomic analysis of S9114 ([GenBank:AFYA01000000], derived from $B$. tianjinese T6-13) and ' $C$. crenatum' MT [GenBank:AQPS01000000] discovered only hundreds of SNPs and InDels (unpublished data). Some of these SNPs and InDels may be responsible for the high glutamate yield. Further genomic analyses should be conducted in a broad range of C. glutamicum strains, especially the parental wild-type strains, to reveal details of their relationships as well as mechanisms for amino acid hyper-production.

\section{Conclusions}

This work has expanded the MLST method to C. glutamicum and developed an efficient technique to discriminate strains of uncertain origin.

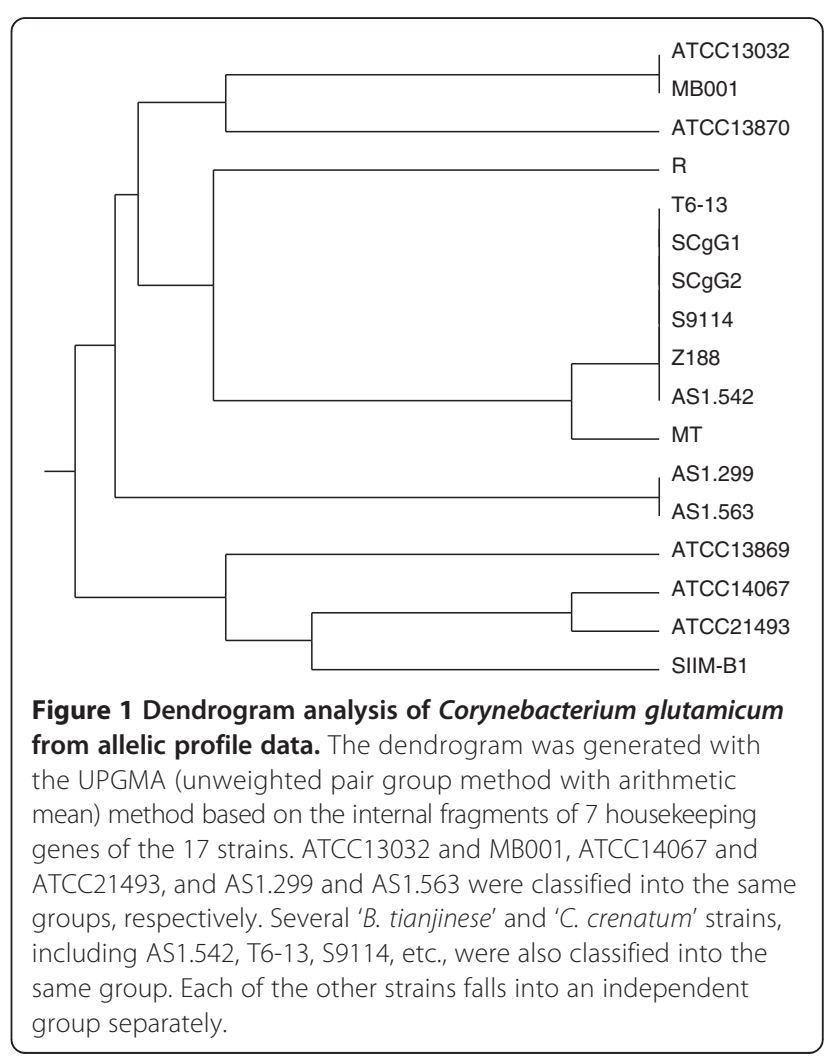




\section{Additional file}

Additional file 1: Supplementary material. Figure S1. PCR fragments of seven genes: atpA 390 bp, dnaE 366 bp, dnaK 397 bp, fusA 402 bp, rpoB $357 \mathrm{bp}$, leuA $318 \mathrm{bp}$, and odhA $327 \mathrm{bp}$. Dataset S1. PCR fragment sequences of seven genes. Dataset S2. Allele sequences of seven genes.

\section{Abbreviations}

MLST: multi-locus sequence typing; ST: sequence type.

\section{Competing interests}

The authors declare that they have no competing interests.

\section{Authors' contributions}

JY and SY designed the research. JY carried out the experiments. JY and YK carried out the computational experiments. JY and YK wrote the manuscript. All authors read and approved the final manuscript.

\section{Acknowledgements}

We thank Prof. Ji-Bin Sun (Tianjin Institute of Industrial Biotechnology, Chinese Academy of Sciences) for providing the draft genome sequences of several strains. This work was supported by a grant from the National Basic Research Program (973 Program) of China (2014CB745100) and the National Key Technologies R\&D Program of China (2012AA022101).

\section{Author details}

'Key Laboratory of Synthetic Biology, Institute of Plant Physiology and Ecology, Shanghai Institutes for Biological Sciences, Chinese Academy of Sciences, 300 Fenglin Road, Shanghai 200032, China. ²Shanghai Research Center of Industrial Biotechnology, Shanghai 201201, China. ${ }^{3}$ University of the Chinese Academy of Sciences, Beijing 100080, China. ${ }^{4}$ Shanghai Collaborative Innovation Center for Biomanufacturing Technology, Shanghai 200237, China

Received: 17 October 2014 Accepted: 12 December 2014

Published online: 23 January 2015

\section{References}

1. Ikeda M, Nakagawa S. The Corynebacterium glutamicum genome: features and impacts on biotechnological processes. Appl Microbiol Biotechnol. 2003:62(2-3):99-109. doi:10.1007/s00253-003-1328-1.

2. Kalinowski J, Bathe B, Bartels D, Bischoff N, Bott M, Burkovski A, et al. The complete Corynebacterium glutamicum ATCC 13032 genome sequence and its impact on the production of L-aspartate-derived amino acids and vitamins. J Biotechnol. 2003;104(1-3):5-25.

3. Liebl W, Ehrmann M, Ludwig W, Schleifer KH. Transfer of Brevibacterium divaricatum DSM 20297T, "Brevibacterium flavum" DSM 20411,

"Brevibacterium lactofermentum" DSM 20412 and DSM 1412, and Corynebacterium glutamicum and their distinction by rRNA gene restriction patterns. Int J Syst Bacteriol. 1991;41(2):255-60.

4. Pascual C, Lawson PA, Farrow JA, Gimenez MN, Collins MD. Phylogenetic analysis of the genus Corynebacterium based on 165 rRNA gene sequences. Int J Syst Bacteriol. 1995;45(4):724-8.

5. Kinoshita S, Nakayama K, Akita S. Taxonomical study of glutamic acid accumulating bacteria, Micrococcus glutamicus nov. sp. Bull Agric Chem Soc Jpn. 1958;22:176-85.

6. Ge B-Z, Wang J-X, Zhu S-J, Si X-D. Identification of glutamic acid producing strains by phages (in Chinese with English abstract). Virol Sin. 1991;6(3):256-9.

7. Bolt F, Cassiday P, Tondella ML, Dezoysa A, Efstratiou A, Sing A, et al. Multilocus sequence typing identifies evidence for recombination and two distinct lineages of Corynebacterium diphtheriae. J Clin Microbiol. 2010;48(11):4177-85. doi:10.1128/JCM. 00274-10.

8. Maiden MC, van Rensburg MJ, Bray JE, Earle SG, Ford SA, Jolley KA, et al. MLST revisited: the gene-by-gene approach to bacterial genomics. Nat Rev Microbiol. 2013;11(10):728-36. doi:10.1038/nrmicro3093.

9. Chen Q, Zhang Z-Y, Li L-G. A new L-glutamic acid-producing species of Corynebacterium (in Chinese with English abstract). Acta Microbiol Sinica (Wei Sheng Wu Xue Bao). 1973;13(1):1-6.

10. Institute_of_Microbiology_Chinese_Academy_of_Sciences, Hangzhou_Glutamate_Factory. Study on the production of lysine by auxotrophic mutant of Corynebacterium pekinense AS1.299 (in Chinese) Microbiol China (Wei Sheng Wu Xue Tong Bao). 1974;1(1):7-11.

11. Chen Q, Li L-G. Studies on L-glutamic acid producing bacteria AS 1.542. I. Identification of strain AS 1.542 (in Chinese with English abstract). Acta Microbiol Sinica (Wei Sheng Wu Xue Bao). 1975;15(2):119-24.

12. Zhang K, Liu Y. Studies on glutamate dehydrogenase from Brevibacterium tianjinese T6-13 (in Chinese with English abstract). Acta Microbiol Sinica (Wei Sheng Wu Xue Bao). 1991:31(4):281-6.

13. Onoda T, Yoshinaga F, Kubota K, Kamijo H, Okumura S (1973) Method of producing l-arginine by microorganism. US Patent 3723249, patent date 27/03/1973

14. Baumgart M, Unthan S, Ruckert C, Sivalingam J, Grunberger A, Kalinowski J, et al. Construction of a prophage-free variant of Corynebacterium glutamicum ATCC 13032 for use as a platform strain for basic research and industrial biotechnology. Appl Environ Microbiol. 2013;79(19):6006-15. doi:10.1128/ AEM. 01634-13.

15. Yukawa H, Omumasaba CA, Nonaka H, Kos P, Okai N, Suzuki N, et al. Comparative analysis of the Corynebacterium glutamicum group and complete genome sequence of strain R. Microbiology. 2007;153(Pt 4):1042-58. doi:10.1099/mic.0.2006/003657-0.

16. LV Y, Liao J, Wu Z, Han S, Lin Y, Zheng S. Genome sequence of Corynebacterium glutamicum ATCC 14067, which provides insight into amino acid biosynthesis in coryneform bacteria. J Bacteriol. 2012;194(3):742-3. doi:10.1128/JB.06514-11

17. LV Y, Wu Z, Han S, Lin Y, Zheng S. Genome sequence of Corynebacterium glutamicum S9114, a strain for industrial production of glutamate. J Bacteriol. 2011;193(21):6096-7. doi:10.1128/JB.06074-11.

18. Jolley KA, Maiden MC. BIGSdb: scalable analysis of bacterial genome variation at the population level. BMC Bioinformatics. 2010;11:595. doi:10.1186/1471-2105-11-595.

19. Xu M, Rao Z, Dou W, Xu Z. The role of ARGR repressor regulation on L-arginine production in Corynebacterium crenatum. Appl Biochem Biotechnol. 2013;170(3):587-97. doi:10.1007/s12010-013-0212-4.

20. Yun F, Zhou W (1994) Breeding and application of a strain of high glutamic

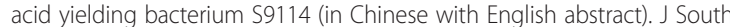
China University of Technol (Natural Science). 1994;22(1):56-62.

\section{Submit your manuscript to a SpringerOpen ${ }^{\odot}$ journal and benefit from:}

- Convenient online submission

- Rigorous peer review

- Immediate publication on acceptance

- Open access: articles freely available online

- High visibility within the field

- Retaining the copyright to your article

Submit your next manuscript at $>$ springeropen.com 